\title{
Lepton Mixing, Residual Symmetries, and Trigonometric Diophantine Equations
}

\author{
Bo $\mathrm{Hu} *$ \\ Department of Physics, Nanchang University, Nanchang 330031, China
}

\begin{abstract}
In this paper, we study residual symmetries in the lepton sector. Our first concern is the symmetry of the charged lepton mass matrix in the basis where the Majorana neutrino mass matrix is diagonal, which is strongly constrained by the requirement that the symmetry group generated by residual symmetries is finite. In a recent work R. M. Fonseca and W. Grimus found that there exists a set of constraint equations that can be completely solved, which is essential in their approach to the classification of lepton mixing matrices that are fully determined by residual symmetries. In this paper, a method to handle trigonometric Diophantine equations is introduced. We will show that the constraint equations found by Fonseca and Grimus can also be solved by this method. Detailed derivation and discussion will be presented in a self-contained way. In addition, we will also show that, in the case where residual symmetries satisfy a reality condition, this method can be used to solve the equation constraining parameters in the symmetry assignment that controls the group structure generated by residual symmetries and is directly related to mixing matrix elements.
\end{abstract}

*Electronic address: bohu@ncu.edu.cn 


\section{INTRODUCTION}

In recent years the relation between lepton mixing and residual symmetries originating from finite flavor groups has received a fair amount of attention [1 12]. Other aspects of residual symmetries have also been studied [13]. Although the idea that lepton mixing may originate from an underlying discrete symmetry has been discussed extensively in literature (for some recent reviews, see, e.g., [14]), often only specific residual symmetries are involved, e.g., in lepton flavor models (see, e.g., [15-18] for models based on various discrete groups including $\mathbf{A}_{4}, \mathbf{S}_{4}, \boldsymbol{\Delta}\left(6 n^{2}\right)$, etc.). With the help of the computer algebra system GAP [19], scans of finite groups have also been performed by several groups to look for viable discrete flavor symmetries [20]. Hence, it is worthwhile to see whether there are any general and model-independent results that can be obtained for residual symmetries and the corresponding flavor symmetries and mixing patterns.

In [6] and [7] Hernandez and Smirnov showed that, if residual symmetries generate a finite group, then the mixing matrix elements can be related to parameters in the symmetry assignment, including several characteristic parameters of residual symmetries. In [8], this method was used to construct full-mixing matrices, i.e., the mixing matrices that are fully determined by residual symmetries. In particular, using an algebraic method to solve the unitary condition which must be satisfied by any symmetry assignment that can generate a full-mixing matrix, we found that all the full-mixing patterns can be determined in the minimal scenario where residual symmetries satisfy a reality condition. In more general cases, explicit expressions for the mixing matrix elements can be obtained (see, e.g., [7] and [8]) and, to obtain specific solutions, algebraic methods can still be useful, but the problem of finding the complete set of solutions becomes more involved. Nevertheless, a clear and complete answer to this problem certainly can improve our understanding of the role that residual symmetries might play.

Remarkably, a complete classification of lepton mixing matrices from residual symmetries was obtained by Fonseca and Grimus in a recent work [12]. Their approach is also based on the assumption that residual symmetries are originated from a finite flavor group. Nevertheless, in this approach, mixing matrices are not obtained from symmetry assignments, but from $T$, the generator of the residual symmetry in the charged lepton sector. Therefore, it is crucial to find explicitly all the possible forms that $T$ can assume. In [12], this is done 
by first establishing the constraint equations of $\left|T_{i j}\right|$, and then showing that these equations can be solved completely by using some mathematical results concerning roots of unity.

In this paper, we will show that those constraint equations of $\left|T_{i j}\right|$ can also be solved by a method developed in section III. Briefly speaking, our method is based on the observation that the equations to be solved can be written as trigonometric Diophantine equations which can be transformed to arithmetic equations involving only rational integers. They are not equivalent, but the latter can be used as auxiliary conditions which may lead to great simplification. The transformation formula can be written in a simple form and will be derived in a self-contained way. Some mathematical details are provided in the appendix.

The solutions to the constraint equations of $\left|T_{i j}\right|$ are derived in Sec. IV by using the method introduced in section III. We will show that these equations admit only a few solutions from which the basic forms of $|T|$ can be obtained. Another use of trigonometric algebraic numbers will also be discussed. In Sec. V, we will show that this method can also be used to find the complete solution of the unitary condition in the minimal scenario. The results were already given and discussed in [8], but the derivation was omitted in order to avoid being distracted by mathematical details ${ }^{1}$ and hence the derivation presented in Sec. V can complete the discussion of the minimal scenario in [8]. We hope that the discussions in this paper and those in other works including [8], [9], and [12] may draw attention to some algebraic notions and techniques that are much less well-known than group theoretical techniques but may also be useful in the discussions of discrete flavor symmetries.

Before proceeding to the main discussion, to provide the necessary background and set our notations, in Sec. II we briefly review and discuss the two approaches mentioned above.

\section{LEPTON MIXING AND RESIDUAL SYMMETRIES}

We denote the generator of the symmetry of the left-handed charged lepton mass matrix $M_{l} M_{l}^{\dagger}$ by $T$ and those of the neutrino mass matrix $M_{\nu}$ by $S_{i}$ where $i=1,2$, or 3 . The symmetry groups generated by $T$ and $S_{i}$ are denoted by $G_{e}$ and $G_{\nu}$, respectively. As usual we adopt the assumption that neutrinos are Majorana particles and $G_{\nu}$ is the Klein four-group:

$$
G_{\nu}=\left\{\mathbf{1}, S_{1}, S_{2}, S_{3}\right\}
$$

\footnotetext{
${ }^{1}$ Some discussions in this paper can also be found in the first arXiv version of [8].
} 
In the basis where $M_{\nu}$ is diagonal, $S_{i}$ can be written as

$$
S_{1}^{d}=\operatorname{diag}\{1,-1,-1\}, \quad S_{2}^{d}=\operatorname{diag}\{-1,1,-1\}, \quad S_{3}^{d}=S_{1}^{d} S_{2}^{d}
$$

On the other hand, in the basis where $M_{l}$ is diagonal, $T$ becomes diagonal and will be denoted by $T_{d}$.

As emphasized in the introduction, the following discussion is based on the assumption that $T$ and $S_{i}$ together generate a finite group $G$, which may be identified with the flavor group $G_{f}$. A necessary condition for this assumption to hold is given by the relations [6]

$$
S_{i}^{2}=T^{m}=\left(W_{i}\right)^{p_{i}}=\mathbb{I}
$$

where $m$ and $p_{i}$ are integers and $W_{i} \equiv\left(S_{i} T\right)^{-1}$. Since the lepton mixing matrix $U=U_{l}^{\dagger} U_{\nu}$ where $U_{l}$ and $U_{\nu}$ are the matrices diagonalizing $T$ and $S_{i}$, then from Eq. (2) one finds that $\left|U_{\rho i}\right|$ can be determined by parameters in the so-called symmetry assignment including $m$ and $p_{i}$. To see that, let us begin with $\operatorname{Tr}\left[W_{i}\right]$. From the definition of $W_{i}$ it follows that

$$
\left(\operatorname{Tr}\left[W_{i}\right]\right)^{*}=\operatorname{Tr}\left[S_{i} T\right]=\operatorname{Tr}\left[U S_{i}^{d} U^{\dagger} T_{d}\right]=2 \operatorname{Tr}\left[A_{i} T_{d}\right]-\operatorname{Tr}\left[T_{d}\right]
$$

where

$$
A_{i}=\operatorname{diag}\left\{\left|U_{1 i}\right|^{2},\left|U_{2 i}\right|^{2},\left|U_{3 i}\right|^{2}\right\} .
$$

In general, because $T^{m}=\left(W_{i}\right)^{p_{i}}=\mathbb{I}$, one can write

$$
T_{d}=\operatorname{diag}\left\{e^{i 2 \pi k_{1} / m}, e^{i 2 \pi k_{2} / m}, e^{i 2 \pi k_{3} / m}\right\}, \quad \operatorname{Tr}\left[W_{i}\right]=\sum_{j=1}^{3} e^{i 2 \pi n_{j} / p_{i}} .
$$

Then from Eqs. (3) and (44) the relations between $\left|U_{\rho i}\right|$ and the parameters in $T_{d}$ and $\operatorname{Tr}\left[W_{i}\right]$ can be established. More details including the explicit expressions can be found in [6] 6 ] and will not be repeated here except for the minimal scenario discussed in Sec. V.

To obtain full-mixing matrices, we shall require that all $S_{i} \in G_{f}$ because one $S_{i}$ fixes only a column in $|U|$. Then, from Eq. (3) and $\sum_{i=1}^{3} S_{i}^{d}=-\mathbb{I}$, it follows that

$$
\sum_{i=1}^{3}\left(\operatorname{Tr}\left[W_{i}\right]\right)^{*}=-\operatorname{Tr}\left[T_{d}\right]
$$

This equation will be referred to as unitarity condition [8], which must be obeyed by any combination of $S_{i}$ and $T$ that generates a full-mixing matrix ${ }^{2}$.

\footnotetext{
${ }^{2}$ Note that, if $T$ has degenerate eigenvalues, one will need another matrix $T^{\prime}$ to determine $|U|$ fully because only one row in $|U|$ can be obtained from Eq. (3) even if all $S_{i} \in G_{f}$.
} 
As mentioned in the introduction, in [8] attempts were made to find possible full-mixing matrices by solving the unitarity condition given by Eq. (5). It was found that in the minimal scenario where $\operatorname{Tr}\left[T_{\alpha}\right]$ and $\operatorname{Tr}\left[W_{i}\right]$ are real, Eq. (5) can be solved completely and then all the possible mixing patterns can also be obtained from its solutions. The detailed derivation omitted in [8] can be found in section V. In non-minimal scenarios, the unitary condition becomes more involved but less restrictive. Nevertheless, solutions to Eq. (5) are still severely constrained by its algebraic nature, as discussed in [8], which is also implied by the results of numerical calculations. Therefore, it is reasonable to think that it might still be possible to obtain a complete picture of full-mixing patterns under assumptions mentioned above, although solving Eq. (5) alone may not be an efficient way because the relations in Eq. (2) are not sufficient for $S_{i}$ and $T$ to generate a finite group.

This problem is solved by Fonseca and Grimus in a recent work. In [12], they begin with $Y^{(i j)}=T^{\dagger} S_{i} T S_{j}$ which must also have finite orders. Since one can show that $\operatorname{det} Y^{(i j)}=1$ and $\operatorname{Tr}\left[Y^{(i j)}\right]$ are real, it then follows that $Y^{(i j)}$ have eigenvalues $1, \lambda^{(i j)},\left(\lambda^{(i j)}\right)^{*}$, where $\lambda^{(i j)}$ are roots of unity and hence can be written as

$$
\lambda^{(i j)}=e^{i 2 \pi k^{(i j)} / m^{(i j)}}
$$

where $k^{(i j)}$ and $m^{(i j)}$ are coprime. Because $\operatorname{Tr}\left[Y^{(i j)}\right]$ are basis independent, from $\sum_{i=1}^{3} S_{i}^{d}=$ $-\mathbb{I}$, one finds that

$$
\sum_{i=1}^{3} \operatorname{Tr}\left[Y^{(i j)}\right]=\sum_{j=1}^{3} \operatorname{Tr}\left[Y^{(i j)}\right]=1
$$

which can be written as

$$
\begin{aligned}
& \sum_{i=1}^{3}\left(\lambda^{(i j)}+\left(\lambda^{(i j)}\right)^{*}\right)=-2 \\
& \sum_{j=1}^{3}\left(\lambda^{(i j)}+\left(\lambda^{(i j)}\right)^{*}\right)=-2 .
\end{aligned}
$$

In addition, in the basis where $S_{i}$ are diagonal, one can show that

$$
4\left|T_{i j}\right|^{2}=\operatorname{Tr}\left[Y^{(i j)}\right]+1=\lambda^{(i j)}+\left(\lambda^{(i j)}\right)^{*}+2
$$

Therefore, from $\lambda^{(i j)}$ satisfying (17) one can construct the matrix $|T|$ which is defined by $|T|_{i j}=\left|T_{i j}\right|$. It was found in [12] that there exist only five basic forms that $|T|$ can assume. From these basic forms one can derive all the possible full-mixing patterns, because in the basis where $S_{i}$ are diagonal the lepton mixing matrix can be obtained from $T$ alone. For 
instance, for a given $T$ in the diagonal basis of $S_{i}$, one may obtain $T_{d}$ from $\operatorname{Tr}[T]$ and det $[T]$ and then solve Eq. (3) for mixing matrix elements.

In [12], the two equations in (7) are solved by employing a theorem related to roots of unity [21]. Here we note that substituting Eq. (66) into (77) leads to

$$
\begin{aligned}
& \sum_{i=1}^{3} 2 \cos \left(\frac{k^{(i j)}}{m^{(i j)}} 2 \pi\right)=-2, \\
& \sum_{j=1}^{3} 2 \cos \left(\frac{k^{(i j)}}{m^{(i j)}} 2 \pi\right)=-2 .
\end{aligned}
$$

In addition, in the minimal scenario the unitary condition in Eq. (5) can also be written in a similar form, as shown in section $\mathrm{V}$. In the next section, we introduce a method to handle trigonometric Diophantine equations, such as the two equations above. Using this method, we derive the solutions to Eqs. (9) and (10) in Sec. IV.

\section{TRIGONOMETRIC DIOPHANTINE EQUATIONS}

In this section, we consider trigonometric Diophantine equations that can be written as

$$
\sum_{j=1}^{n} 2 \cos \frac{n_{j}}{p_{j}} 2 \pi=r
$$

where $n_{j}, p_{j}$, and $r$ are rational integers. Without loss of generality, we require that $n_{j}$ and $p_{j}$ are coprime or $\operatorname{gcd}\left(n_{j}, p_{j}\right)=1$ where gcd stands for the greatest common divisor. To simplify notations, below we denote rational angles such as $2 \pi n / m$ by $\alpha_{n m}$ and $2 \cos \alpha_{n_{j} p_{j}}$ by $\beta^{j}$. Then Eq. (11) can be written as

$$
\sum_{j=1}^{n} \beta^{j}=\sum_{j=1}^{n} 2 \cos \alpha_{n_{j} p_{j}}=r .
$$

We will show that this equation can be transformed to a simple arithmetic equation ${ }^{3}$, i.e.,

$$
\sum_{j=1}^{n} \frac{s_{j}}{d_{j}}=r
$$

where $d_{j}$ is the degree of the minimal polynomial (MP) of $\beta^{j}$, i.e., the polynomial with integer coefficients satisfied by $\beta^{j}$ that has the lowest degree ${ }^{4}$, and $s_{j}$ is the sum of all the

\footnotetext{
${ }^{3}$ Similar results may exist somewhere in literature, but we are not aware of any of them.

${ }^{4}$ In other words, it is irreducible or cannot be written as a product of two monic polynomials with integer coefficients. More can be found in the appendix.
} 
roots of that MP. Note that not only $d_{j}$, but also $s_{j}$, are rational integers, as explained below. From solutions to Eq. (13) one can find solutions to Eq. (11) because $p_{j}$ can be determined by $d_{j}$ and $s_{j}$. To derive Eq. (13), we will need some notions and results from algebraic number theory, mostly for convenience. The detailed derivation is presented below in a rather self-contained way, but, for conciseness, some details are relegated to the appendix. Before proceed, we should mention that in this paper we only use polynomials with integer coefficients, unless otherwise stated. In addition, the set of all rational numbers and the set of all rational integers are denoted by $\mathbb{Q}$ and $\mathbb{Z}$, respectively.

First, note that from

$$
\cos 2 \pi n_{j}=\cos \left(p_{j} \cdot \alpha_{n_{j} p_{j}}\right)=1
$$

and the expansion of $\cos \left(p_{j} \alpha_{n_{j} p_{j}}\right)$ in powers of $\cos \left(\alpha_{n_{j} p_{j}}\right)$, as shown in the appendix, one can see that $\beta^{j}=2 \cos \alpha_{n_{j} p_{j}}$ are algebraic integers which are defined to be solutions to monic polynomials with integer coefficients. Moreover, all the $\beta^{j}=2 \cos \left(2 \pi n_{j} / p_{j}\right)$ in Eq. (12) can be expanded in powers of

$$
\gamma_{1} \equiv 2 \cos \frac{2 \pi}{q}
$$

where $q \equiv \operatorname{LCM}\left(p_{1}, \ldots, p_{n}\right)$ and LCM stands for the least common multiple. Obviously, $\gamma_{1}$ is also an algebraic integer. Let polynomial $g(x)$ of degree $d$ be the MP for $\gamma_{1}$. It can be written as

$$
g(x)=x^{d}+c_{d-1} x^{d-1}+\cdots+c_{0}=\prod_{i=1}^{d}\left(x-\gamma_{i}\right)
$$

where $\gamma_{i}$ are the roots of $g(x)$. Since the degree of an algebraic integer is defined to be the degree of its MP, we have $\operatorname{deg}\left(\gamma_{1}\right)=d$ where $\operatorname{deg}\left(\gamma_{1}\right)$ denotes the degree of $\gamma_{1}$.

To proceed, we notice that, using $g\left(\gamma_{1}\right)=0$, one can eliminate any term having a power of $\gamma_{1}$ higher than $d-1$ from a polynomial in $\gamma_{1}$. Hence, if an algebraic integer $\theta$ can be written as a polynomial in $\gamma_{1}$, then one can always write $\theta$ as

$$
\theta=a_{0}+a_{1} \gamma_{1}+\cdots+a_{d-1} \gamma_{1}^{d-1}=\sum_{k=0}^{d-1} a_{k}\left(\gamma_{1}\right)^{k} \equiv p\left(\gamma_{1}\right)
$$

where $a_{i} \in \mathbb{Q}$. More importantly, the expression for $\theta$ in the form of Eq. (14) is unique. To show that, suppose $\theta$ can also be written as

$$
\theta=a_{0}^{\prime}+a_{1}^{\prime} \gamma_{1}+\cdots+a_{d-1}^{\prime} \gamma_{1}^{d-1}
$$


Then one has

$$
\left(a_{d-1}^{\prime}-a_{d-1}\right) \gamma_{1}^{d-1}+\cdots+\left(a_{1}^{\prime}-a_{1}\right) \gamma_{1}+\left(a_{0}^{\prime}-a_{0}\right)=0 .
$$

Since the degree of $\gamma_{1}$ is $d$ and thus $\gamma_{1}$ cannot satisfy any polynomial of degree less than $d$, it then follows that $a_{k}^{\prime}=a_{k}$ for all $k$.

According to the discussion above, we can write $\beta^{j}$ uniquely as

$$
\beta^{j}=\sum_{k=0}^{d-1} b_{k}^{j}\left(\gamma_{1}\right)^{k} \equiv \tilde{\beta}^{j}\left(\gamma_{1}\right)
$$

where $b_{k} \in \mathbb{Z}$. Then, from Eqs. (12) and (15) one has

$$
\sum_{j=1}^{n} \tilde{\beta}^{j}\left(\gamma_{1}\right)=\sum_{k=0}^{d-1}\left(\sum_{j=1}^{n} b_{k}^{j}\right)\left(\gamma_{1}\right)^{k}=r .
$$

Now comes a crucial step. Again, since $\gamma_{1}$ cannot satisfy any polynomial of degree less than $d$, in Eq. (16) one must have

$$
\sum_{j=1}^{n} b_{k}^{j}=0
$$

for $1 \leq k \leq d-1$ because $r$ is a rational integer. Hence, in Eq. (16) one may replace $\gamma_{1}$ by any number. For our purpose, we will substitute $\gamma_{1}$ by $\gamma_{2}, \ldots, \gamma_{d}$, i.e., the other roots of its MP. Then, besides Eq. (16), we also have

$$
\sum_{j=1}^{n} \tilde{\beta}^{j}\left(\gamma_{i}\right)=r
$$

for $2 \leq i \leq d$. Summing over $i$ leads to

$$
\sum_{j=1}^{n}\left[\sum_{i=1}^{d} \tilde{\beta}^{j}\left(\gamma_{i}\right)\right]=r d .
$$

In addition, as discussed in the appendix, one can show that for any $\gamma_{i}$ the corresponding $\tilde{\beta}^{j}\left(\gamma_{i}\right)$ is a root of the MP of $\tilde{\beta}^{j}\left(\gamma_{1}\right)$ because $\gamma_{i}$ is a root of the MP of $\gamma_{1}$. Now, let $d_{j}$ be the degree of $\beta^{j}=\tilde{\beta}^{j}\left(\gamma_{1}\right)$. Because one can also show that when $\gamma_{i}$ in $\tilde{\beta}^{j}\left(\gamma_{i}\right)$ runs from $\gamma_{1}$ to $\gamma_{d}$, each root of the MP of $\tilde{\beta}^{j}\left(\gamma_{1}\right)$ repeats $d / d_{j}$ times, we have

$$
\sum_{i=1}^{d} \tilde{\beta}^{j}\left(\gamma_{i}\right)=\frac{d}{d_{j}} s_{j}
$$

where $s_{j}$ is the sum of all the roots of the MP of $\beta^{j}$. From Eqs. (17) and (18) it follows that

$$
\sum_{j=1}^{n} \frac{s_{j}}{d_{j}}=\sum_{j=1}^{n} \frac{1}{\operatorname{deg}\left(\beta^{j}\right)} \sum_{c} \beta^{j}=r
$$


where $\operatorname{deg}\left(\beta^{j}\right)=d_{j}$ and $\quad \sum_{c} \beta^{j}=s_{j}$. We would like to emphasize that $\sum_{c} \beta^{j}$ should not be confused with $\sum_{j} \beta^{j}$. In addition, since the MP of $\beta^{j}$ can be written as

$$
x^{d_{j}}-s_{j} x^{d_{j}-1}+\cdots,
$$

then $s_{j}$ must be a rational integer.

In the appendix, the explicit expressions for $d_{m}=\operatorname{deg}\left(2 \cos \alpha_{n m}\right)$ and $s_{m}=\sum_{c} 2 \cos \alpha_{n m}$ are given in Eq. (A2) and Eq. (A3). As examples, $d_{m}$ and $s_{m}$ for $2 \leq m \leq 20$ are given in the table below, which will also be used in the next two sections. Note that $d_{m}$ and $s_{m}$ together can determine the value of $m$ in $\alpha_{n m}=2 \pi n / m$ but not $n$ which can be any integer that is relatively prime to $m$. For obvious reasons, we can require that $0<n<m / 2$. In addition, the value of $m$ may not be determined uniquely by $d_{m}$ and $s_{m}$, as can be seen from table II.

\begin{tabular}{|l|l|l|l|l|l|l|l|l|l|l|l|l|l|l|l|l|l|l|l|l|}
\hline$m$ & 1 & 2 & 3 & 4 & 5 & 6 & 7 & 8 & 9 & 10 & 11 & 12 & 13 & 14 & 15 & 16 & 17 & 18 & 19 & 20 \\
\hline$d_{m}$ & 1 & 1 & 1 & 1 & 2 & 1 & 3 & 2 & 3 & 2 & 5 & 2 & 6 & 3 & 4 & 4 & 8 & 3 & 9 & 4 \\
\hline$s_{m}$ & 2 & -2 & -1 & 0 & -1 & 1 & -1 & 0 & 0 & 1 & -1 & 0 & -1 & 1 & 1 & 0 & -1 & 0 & -1 & 0 \\
\hline
\end{tabular}

TABLE I: The degree of the MP of $2 \cos \alpha_{n m}$ and the sum of its roots

The issues mentioned above indicate that Eq. (19) is only a necessary condition for any set of $p_{j}$ or, more accurately, $\beta^{j}=2 \cos \left(2 \pi n_{j} / p_{j}\right)$ to satisfy Eq. (11). Although it is not a sufficient condition and hence some solutions to Eq. (19) may not satisfy Eq. (11), because it is not hard to find its solutions in many cases, Eq. (19) can be regarded as an auxiliary condition which may greatly simplify the problem of finding solutions to Eq. (11). As to $n_{j}$ in Eq. (11), one can find them by trial and error, especially when $d_{j}$ is small because, as explained in the appendix, $d_{j}$ is the number of $n_{j} \operatorname{satisfying} \operatorname{gcd}\left(n_{j}, p_{j}\right)=1$ and $0<n_{j}<p_{j} / 2$.

Eq. (19) is most helpful in the cases where it can provide enough information for us to solve Eq. (11) completely. Such situation may occur if the number of terms on the left-hand side or $n$ is not large and $|r|$ is comparable to $n$. The reason is that $s_{j}=\sum_{c} 2 \cos \left(2 \pi n_{j} / p_{j}\right)$ can only be \pm 1 or 0 for all $p_{j}$ (except for $p_{j}=1$ or 2 ) and the lower bound on $d_{j}$ increases with $p_{j}$ (see the discussion below Eq. A2 in the appendix). Hence, for a small $n$ and a $|r| \sim n$, to satisfy Eq. (19), $p_{j}$ cannot all be very large in most cases and thus it might be 
possible to solve it completely. This is exactly the situation for the two cases discussed in the following two sections. This method may also be useful in some other cases, for example, when one only wants to find certain specific solutions or for some reason $p_{j}$ can only be chosen from a given set of numbers.

\section{RESIDUAL SYMMETRY IN THE CHARGED LEPTON SECTOR}

In this section, we will show that the complete set of solutions to Eqs. (9) and (10) can be easily obtained by the method introduced above. Since these two equations are similar, only Eq. (9) will be discussed in detail. As above, to simplify notations, we write Eq. (9) as

$$
\sum_{i=1}^{3} \beta^{(i j)}=\sum_{i=1}^{3} 2 \cos \alpha^{(i j)}=-2
$$

where $\alpha^{(i j)}=2 \pi k^{(i j)} / m^{(i j)}$. Then, as shown in the previous section, this equation can be transformed to

$$
\sum_{i=1}^{3} y_{i}=\sum_{i=1}^{3} \frac{s_{i}}{d_{i}}=\sum_{i=1}^{3} \frac{1}{\operatorname{deg}\left(\beta^{(i j)}\right)} \sum_{c} \beta^{(i j)}=-2
$$

where $y_{i}=s_{i} / d_{i}, s_{i}=\sum_{c} \beta^{(i j)}$, and $d_{i}=\operatorname{deg}\left(\beta^{(i j)}\right)$.

Since, as discussed above, $\left|s_{i}\right| \leq 1$ for $m^{(i j)}>2$ and $d_{i}$ tend to grow with $m^{(i j)}$, one can show that

$$
\left|\frac{s_{j}}{d_{j}}\right| \leq 2
$$

for any $j$. Hence, without loss of generality, we can require that

$$
2 \geq\left|y_{1}\right| \geq\left|y_{2}\right| \geq\left|y_{3}\right|
$$

Then from Eq. (21) it follows that

$$
\left|y_{1}\right|=\left|\frac{s_{1}}{d_{1}}\right| \geq \frac{2}{3}
$$

and hence in table 1 one finds that

$$
m^{(1 j)}=1,2,3, \text { or } 6 .
$$

If $m^{(1 j)}=1$, then

$$
\alpha^{(1 j)}=2 \pi, \quad \beta^{(1 j)}=2 \cos \alpha^{(1 j)}=2 .
$$


Substituting $\beta^{(1 j)}=2$ into Eq. (20) leads to

$$
2 \cos \alpha^{(2 j)}+2 \cos \alpha^{(3 j)}=-4 .
$$

Therefore, $m^{(2 j)}=m^{(3 j)}=2$ and hence

$$
\beta^{(2 j)}=\beta^{(3 j)}=2 \cos \left(\frac{1}{2} \times 2 \pi\right)=-2 .
$$

In the case where $m^{(1 j)}=2$, one has $\alpha^{(1 j)}=\pi$ and

$$
\beta^{(1 j)}=2 \cos \alpha^{(1 j)}=-2
$$

Then from Eq. (20) it follows that

$$
\beta^{(2 j)}=-\beta^{(3 j)}=2 \cos \varphi
$$

where $\varphi=\alpha^{(2 j)}$ is an arbitrary rational angle. Note that, no further constraint can be imposed on $\varphi$ in this case because Eq. (27) can be satisfied if

$$
\frac{k^{(2 j)}}{m^{(2 j)}}+\frac{k^{(3 j)}}{m^{(3 j)}}=\frac{1}{2}
$$

from which one can find a $m^{(3 j)}$ for any $m^{(2 j)}$.

The next case is the one where $m^{(1 j)}=3$, which is a bit more complicated. From table 1. one finds that $d_{1}=1$ and $s_{1}=-1$ and hence $y_{1}=-1$. Then from Eq. (21), one has $y_{2}+y_{3}=-1$. Together with Eq. (23) and $\left|y_{1}\right|=1$, this leads to $1 / 2 \leq\left|y_{2}\right| \leq 1$ and $\left|y_{3}\right| \leq 1$. From these results one finds that $-1 \leq y_{2} \leq-1 / 2$. Therefore, $y_{2}=-1$ or $-1 / 2$, as can be seen from table II. If $y_{2}=-1$, one has $m^{(2 j)}=3$. Together with $m^{(1 j)}=3$ and Eq. (201), it leads to $\beta^{(3 j)}=0$ and hence $m^{(3 j)}=4$. In short, if $y_{2}=-1$, one has

$$
\begin{aligned}
& \beta^{(1 j)}=\beta^{(2 j)}=2 \cos \frac{2 \pi}{3}=-1, \\
& \beta^{(3 j)}=2 \cos \frac{2 \pi}{4}=0 .
\end{aligned}
$$

If $y_{2}=-1 / 2$, then $y_{3}=-1 / 2$. From table凹, one has $m^{(2 j)}=m^{(3 j)}=5$. Because $d_{2}=d_{3}=2$, in Eq. (20) both $k^{(2 j)}$ and $k^{(3 j)}$ have two choices. The solution to Eq. (20) is found to be $k^{(2 j)}=1$ and $k^{(3 j)}=2$. Therefore, one has

$$
\begin{aligned}
& \beta^{(1 j)}=2 \cos \frac{2 \pi}{3}=-1, \\
& \beta^{(2 j)}=2 \cos \frac{2 \pi}{5}, \quad \beta^{(3 j)}=2 \cos \frac{4 \pi}{5} .
\end{aligned}
$$


After that, we are left with the case where $m^{(1 j)}=6$. In table $\Pi$ one finds that $d_{1}=1$ and $s_{1}=1$. However, from Eq. (21) it follows that $y_{2}+y_{3}=-3$ which is not consistent with Eq. (23), since the latter would lead to $\left|y_{2}+y_{3}\right| \leq 2\left|y_{1}\right|=2$. Therefore, no solution satisfying Eq. (23) exists for $m^{(1 j)}=6$.

Now, we have found all the solutions to Eq. (20) which are given by Eqs. (24) 29 ). Since, from Eqs. (66) and (8) one has

$$
4\left|T_{i j}\right|^{2}=\beta^{(i j)}+2=2 \cos \left(\frac{k^{(i j)}}{m^{(i j)}} 2 \pi\right)+2,
$$

then, for each solution, the corresponding $\left|T_{i j}\right|^{2}$ can be obtained from the relation above and written collectively as $\left(\left|T_{1 j}\right|^{2},\left|T_{2 j}\right|^{2},\left|T_{3 j}\right|^{2}\right)$. For the four solutions found above, one has the following four possibilities:

$$
\begin{aligned}
& (1,0,0), \quad\left(\frac{1}{4}, \frac{3+\sqrt{5}}{8}, \frac{3-\sqrt{5}}{8}\right) \\
& \left(\frac{1}{2}, \frac{1}{4}, \frac{1}{4}\right), \quad\left(0, \frac{1+\cos \varphi}{2}, \frac{1-\cos \varphi}{2}\right) .
\end{aligned}
$$

Note that the order of $\left|T_{i j}\right|^{2}$ can be changed because in Eq. (23) the order of $y_{1}, y_{2}$, and $y_{3}$ can also be changed, which may lead to solutions with different orderings.

It is easy to see that the solutions to the second equation in (17) are the same as the first one. Hence, in matrix $|T|^{2}$ where $\left(|T|^{2}\right)_{i j}=\left|T_{i j}\right|^{2}$ every row and column must be one of the four possibilities given above. It is then not hard to find that there exist only five basic forms that $|T|$ can assume, as shown in [12]. After that, by a thorough and careful analysis, which can also be found in [12], one can find all the possible full-mixing patterns.

Before proceeding to the next section, we would like to mention that some results used in our discussions might also be useful on other occasions. For example, because a non-integer rational number is not an algebraic integer, as shown in the appendix, sometimes this can provide a quick way to show that for a given complex number $\eta$ there does not exist a rational integer $m$ satisfying $\eta^{m}=1$. If such a $m$ exists, then $\eta$ is a root of unity and can be written as $\eta=e^{i 2 \pi k / m}$. Hence $\eta+\eta^{*}=2 \cos (2 \pi k / m)$ must be an algebraic integer. But, if $\eta+\eta^{*}$ is a non-integer rational number, then it cannot be an algebraic integer and thus $\eta$ cannot be a root of unity. For instance, $\eta=(1+i 3 \sqrt{7}) / 8$ or $\eta=(-1+i \sqrt{15}) / 4$ cannot be a root of unity because $\eta+\eta^{*}$ is a non-integer rational number ${ }^{5}$.

\footnotetext{
${ }^{5}$ These two examples are taken from [12] where a somewhat different argument is used.
} 


\section{MIXING PATTERNS IN THE MINIMAL SCENARIO}

The derivation of lepton mixing patterns from $|T|$ is rather complicated. In this section, we will show that, in the minimal scenario where both $\operatorname{Tr}[T]$ and $\operatorname{Tr}\left[W_{i}\right]$ are real, the unitarity condition (5) can also be solved completely and hence full-mixing matrices can be obtained in a rather straightforward way.

When $\operatorname{Tr}[T]$ is real and $T$ belongs to $S U(3)$, from Eq. (2) it follows that $T_{d}$ can be written as one of the following three matrices:

$$
\begin{aligned}
& T_{e} \equiv T_{1}=\operatorname{diag}\left\{1, e^{2 \pi i n_{4} / p_{4}}, e^{-2 \pi i n_{4} / p_{4}}\right\} \\
& T_{\mu} \equiv T_{2}=\operatorname{diag}\left\{e^{2 \pi i n_{4} / p_{4}}, 1, e^{-2 \pi i n_{4} / p_{4}}\right\} \\
& T_{\tau} \equiv T_{3}=\operatorname{diag}\left\{e^{2 \pi i n_{4} / p_{4}}, e^{-2 \pi i n_{4} / p_{4}}, 1\right\}
\end{aligned}
$$

where $n_{4}$ and $p_{4}$ are coprime. It then follows that

$$
\operatorname{Tr}[T]=1+2 \cos 2 \pi n_{4} / p_{4}
$$

In this section, we will denote $T_{d}$ by $T_{\alpha}$ where $\alpha=1,2$, or 3 . In addition, we require that $m>2$, because otherwise the lepton mixing matrix cannot be fully determined. Similarly, if $\operatorname{Tr}\left[W_{i}\right]$ is real, because $\left(W_{i}\right)^{p_{i}}=\mathbb{I}$, the eigenvalues of $W_{i}$ can always be written as $1, e^{2 \pi i n_{i} / p_{i}}$, or $e^{-2 \pi i n_{i} / p_{i}}$ with $\operatorname{gcd}\left(n_{i}, p_{i}\right)=1$. Hence,

$$
\operatorname{Tr}\left[W_{i}\right]=1+2 \cos 2 \pi n_{i} / p_{i}
$$

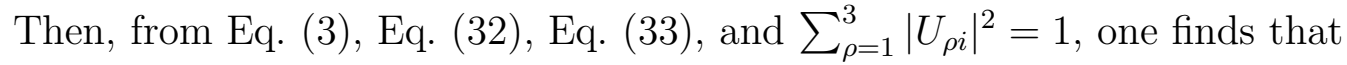

$$
\begin{aligned}
& \left|U_{\alpha i}\right|^{2}=\frac{1+\operatorname{Tr}\left[W_{i}\right]}{3-\operatorname{Tr}\left[T_{\alpha}\right]}=\frac{1+\cos \left(2 \pi n_{i} / p_{i}\right)}{2 \sin ^{2}\left(\pi n_{4} / p_{4}\right)} \\
& \left|U_{\beta i}\right|^{2}=\left|U_{\gamma i}\right|^{2}=\frac{1}{2}\left(1-\left|U_{\alpha i}\right|^{2}\right)
\end{aligned}
$$

where $\beta, \gamma \neq \alpha$ and $\beta<\gamma$. For detailed derivation, see [6] .

As discussed in Sec. II, to generate a full-mixing matrix, $W_{i}$ and $T_{\alpha}$ must satisfy the unitary condition in Eq. (5). In the minimal scenario, substituting Eqs. (32) and (33) into Eq. (15) leads to

$$
\sum_{j=1}^{4} \beta^{j}=\sum_{j=1}^{4} 2 \cos \alpha_{n_{j} p_{j}}=-4
$$


where $\beta^{j}=2 \cos \alpha_{n_{j} p_{j}}$ and $\alpha_{n_{j} p_{j}}=2 \pi n_{j} / p_{j}$. As in the previous section, this equation can be transformed to

$$
\sum_{j=1}^{4} y_{j}=\sum_{j=1}^{4} \frac{s_{j}}{d_{j}}=\sum_{j=1}^{4} \frac{1}{\operatorname{deg}\left(\beta^{j}\right)} \sum_{c} \beta^{j}=-4
$$

which can also be easily solved in a way similar to the previous section.

First, note that Eq. (37) cannot be satisfied if all $y_{j}>-1$, then we can require that $y_{1} \leq-1$ and $y_{1} \leq y_{j}$ for $j \geq 2$. From table【 one finds that $y_{1}=-1$ or -2 corresponding to $d_{1}=1$ and $s_{1}=-1$ or -2 .

If $d_{1}=1$ and $s_{1}=-1$, from Eq. (37) it follows that $\sum_{j=2}^{4} y_{j}=-3$. Since $y_{j} \geq y_{1}=-1$ for $j \geq 2$, it cannot be satisfied unless all $y_{j}=-1$. Therefore, from table $\Pi$ one finds that $s_{j}=-1$ and $d_{j}=1$ and hence $p_{j}=3$ for all $j$.

If $d_{1}=1$ and $s_{1}=-2$, from table $\square$ one finds that $p_{1}=2$. Since one can only let $n_{1}=1$, from Eq. (36) it follows that $\sum_{j=2}^{4} \beta^{j}=-2$. Besides that, from Eq. (37) and $y_{1}=-2$ one has $\sum_{j=2}^{4} y_{j}=-2$. These equations are identical to Eqs. (20) and (21) and hence their solutions can be obtained from those found in Sec. IV.

Here we denote the solutions to Eq. (36) by $\left\{p_{1}, p_{2}, p_{3}, p_{4}\right\}$ from which the corresponding mixing matrix can be obtained from Eqs. (34) and (35). In this notation the solutions we found can be written as $\{3,3,3,3\},\{2,1,2,2\},\left\{2,2, p_{3}, p_{4}\right\},\{2,3,3,4\}$ and $\{2,3,5,5\}$. As explained in the previous section, in the third solution, $p_{3}$ and $p_{4}$ can be any integers that satisfy the relation $n_{3} / p_{3}+n_{4} / p_{4}=1 / 2$ where $n_{3}$ and $n_{4}$ are arbitrary integers satisfying $0<n_{j} / p_{j} \leq 1 / 2$ and $\operatorname{gcd}\left(n_{j}, p_{j}\right)=1$. In Eq. (37) one may change the order of $y_{j}$ and hence

the order of $p_{j}$ can also be changed in these solutions. Nevertheless, as discussed in [8], only the last two solutions can lead to phenomenologically interesting mixing patterns including TBM, BM, or the golden ratio mixings. For more discussion, see [8].

\section{DISCUSSIONS}

In the previous two sections, under the assumption that residual symmetries are originated from a finite group, we discussed the condition constraining the symmetry of the charged lepton mass matrix in the basis where the neutrino mass matrix is diagonal and the unitary condition in the minimal scenario. Both of them can be completely solved by the algebraic method presented in section III and their solutions can be used to construct 
full-mixing matrices. This method can also be used to solve trigonometric Diophantine equations similar to those discussed in this paper.

It is interesting to see that, besides group theoretical techniques, other algebraic techniques can also be useful and even the key to some important results including the basic forms of $|T|$ discussed in section IV. As another example, in [12] it was found that, under the same assumptions adopted in this paper, only particular trimaximal mixing patterns can survive the current neutrino oscillation data. The corresponding symmetry groups include, e.g., $\boldsymbol{\Delta}(600), \boldsymbol{\Delta}(1536)$, and $\left(\mathbf{Z}_{18} \times \mathbf{Z}_{6}\right) \rtimes \mathbf{S}_{3}$, which can also be obtained from solutions to Eq. (5) in non-minimal scenarios. To find those solutions, notions and tools from algebraic number theory can also be helpful, as discussed in [8]. In addition, from Eq. (44) one finds that the unitary condition in Eq. (5) can be written as a vanishing sum of 12 roots of unity. The classification of its solutions can be found in [28] from which one may find another approach to the classification of full-mixing patterns. Hopefully, the works presented in some papers including [9], [12], [8], and this one may draw attention to those less well-known techniques.

Acknowledgements - This work was supported in part by the National Science Foundation of China (NSFC) under the grant 10965003. It was also partly supported by 555 talent project of Jiangxi Province.

\section{Appendix A: Trigonometric algebraic numbers}

In this appendix, some mathematical details concerning trigonometric algebraic numbers will be provided. Below we first quickly recall some basic notions from algebraic number theory [22]:

1. An algebraic number $\theta$ over $\mathbb{Q}$, the set (or field) of all rational numbers, is a root of a monic polynomial over $\mathbb{Q}$ which can be written as

$$
f(x)=x^{n}+a_{n-1} x^{n-1}+\ldots+a_{0}
$$

where all $a_{i} \in \mathbb{Q}$ and $n$ is called the degree of the polynomial. Moreover, if all $a_{i}$ are rational integers, $\theta$ can also be called an algebraic integer. If $\alpha$ and $\beta$ are algebraic numbers (integers), then $\alpha+\beta$ and $\alpha \beta$ are also algebraic numbers (integers). 
Note that, unless otherwise mentioned, here we consider only algebraic numbers (integers) and polynomials over $\mathbb{Q}$, as defined above. Hence, from now on we will omit the phrase "over $\mathbb{Q}$ " for the sake of conciseness.

2. The minimal polynomial (MP) of $\theta$ is the polynomial of lowest degree that $\theta$ satisfies. One can show that the MP of $\theta$ is unique, otherwise one can construct a polynomial satisfied by $\theta$ of lower degree than the MP. For the same reason, the MP is clearly irreducible or cannot be written as a product of two polynomials.

3. The degree of $\theta$, denoted by $\operatorname{deg}(\theta)$, is defined to be the degree of its MP. All the roots of its MP are also called the conjugates of $\theta$, which are denoted by $\theta_{k}$ where $k=1,2, \ldots, \operatorname{deg}(\theta)$ and $\theta_{1}=\theta$. An important and frequently used result is that any polynomial satisfied by $\theta$ must be divisible by its MP and hence satisfied by all $\theta_{k}$ because the remainder of the division, if not vanishing, is also satisfied by $\theta$, but of lower degree than its MP. As a consequence, the MP of $\theta$ is also the MP of $\theta_{k}$ since it is not reducible.

4. Since the MP of an algebraic number (integer) $\theta$ has rational (integer) coefficients and can always be written as $\prod_{k}\left(x-\theta_{k}\right)$, it is obvious that $\sum_{k} \theta_{k}$ is a rational number (integer). One can also show that $\theta_{k}$ are distinct because, if $\theta_{i}=\theta_{j}$, then from the MP one can construct a polynomial satisfied by $\theta_{i}$ or $\theta_{j}$ of lower degree than the MP by taking derivative with respect to $x$.

Mostly for convenience, simple algebraic extension of $\mathbb{Q}$ is introduced below. No deep result concerning field extensions is needed for our discussions.

5. By adjoining to $\mathbb{Q}$ an algebraic number $\theta$, the field of rational numbers can be extended to another field denoted by $\mathbb{Q}(\theta)$. As shown in Sec. III, every element $\lambda$ of $\mathbb{Q}(\theta)$ can be written uniquely in the form

$$
\lambda=a_{0}+a_{1} \theta+\cdots+a_{v-1} \theta^{d-1} \equiv p(\theta)
$$

where $a_{i} \in \mathbb{Q}$ and $d=\operatorname{deg}(\theta)$. The conjugates of $\lambda$ for $\mathbb{Q}(\theta)$ are defined to be $p\left(\theta_{k}\right) \equiv \bar{\lambda}_{k}$ where $\theta_{k}$ are the conjugates of $\theta$ and $\bar{\lambda}_{1}=\lambda$. 
We should emphasize that $\bar{\lambda}_{i}$ defined above are the conjugates of $\lambda$ for $\mathbb{Q}(\theta)$, but not the conjugates of $\lambda$ over $\mathbb{Q}$ (defined in item 3), which are denoted by $\lambda_{k}$. Nevertheless, they are closely related:

6. The set $\left\{\bar{\lambda}_{1}, \bar{\lambda}_{2}, \ldots, \bar{\lambda}_{d}\right\}$ is not necessarily identical to the set of the conjugates of $\lambda$ over $\mathbb{Q}$, i.e., $\left\{\lambda_{1}, \lambda_{2}, \ldots, \lambda_{\operatorname{deg}(\lambda)}\right\}$ where $\lambda_{1}=\lambda=\bar{\lambda}_{1}$. However, one can show that in $\left\{\bar{\lambda}_{1}, \bar{\lambda}_{2}, \ldots, \bar{\lambda}_{d}\right\}$ each $\lambda_{k}$ repeats $d / \operatorname{deg}(\lambda)$ times.

It might be instructive to go over the proof of the result given in item 6 , which is very important in the derivation of Eq. (13). Below we follow [22]. To begin with, one consider the polynomial ${ }^{6}$

$$
f(x)=\prod_{i=1}^{d}\left(x-\bar{\lambda}_{i}\right)=\prod_{i=1}^{d}\left(x-p\left(\theta_{i}\right)\right) .
$$

Let the MP of $\lambda$ be $g(x)$ of degree $\operatorname{deg}(\lambda)$. Since $\lambda=\bar{\lambda}_{1}$ is a root of $f(x)$, then, for the reason explained in item $3, f(x)$ must be divisible by $g(x)$. Therefore, one can write

$$
f(x)=[g(x)]^{n} h(x)
$$

where $g(x) \nmid h(x)$. Then one can show that $h(x)$ must be a constant. Otherwise, for some $\bar{\lambda}_{m}$, one must have $h\left(\bar{\lambda}_{m}\right)=h\left(p\left(\theta_{m}\right)\right)=0$. Since $\theta_{m}$ satisfies $h(p(x))$, then $h(p(x))$ must also be satisfied by $\theta_{1}$ (see item 3 ). Therefore, one has $h\left(p\left(\theta_{1}\right)\right)=h\left(\bar{\lambda}_{1}\right)=h(\lambda)=0$ from which it follows that $g(x) \mid h(x)$ which contradicts the requirement $g(x) \nmid h(x)$. After that, it is not hard to see that $h(x)=1$ and hence $f(x)=[g(x)]^{n}$ and $n=d / \operatorname{deg}(\lambda)$.

Below we will concentrate on trigonometric algebraic numbers, especially the cosines of rational angles, i.e., $2 \cos \alpha_{n m}$ where $\alpha_{n m} \equiv 2 \pi n / m$ and $\operatorname{gcd}(n, m)=1$. As in the main text, below we denote $2 \cos \alpha_{n m}$ by $\beta_{n m}$.

First of all, recall that [23]

$$
2 \cos m \alpha=(2 \cos \alpha)^{m}-m(2 \cos \alpha)^{m-2}+\frac{m(m-3)}{2}(2 \cos \alpha)^{m-4}+\cdots .
$$

\footnotetext{
${ }^{6}$ One can show that the coefficients of $f(x)$ are rational numbers or integers because they can be written as polynomials of the elementary symmetric functions in $\theta_{1}, \ldots \theta_{k}$ such as $\sum_{k} \theta_{k}, \sum_{k, j} \theta_{k} \theta_{j}$, etc., which can be shown to be rational numbers or integers since they are the coefficients of the MP of $\theta_{k}$, i.e., $\prod_{k}\left(x-\theta_{k}\right)$. A complete proof of this point would take us too far afield. One may find the details in [22].
} 
Replacing $2 \cos \alpha$ by $x$ in the expression on the right-hand side, we can define polynomials $c_{m}(x)$ as $^{7}$

$$
c_{m}(x) \equiv\left[x^{m}-m x^{m-2}+\frac{m(m-3)}{2} x^{m-4}+\cdots\right]-2 .
$$

From $\cos m \alpha_{n m}=1$, one has

$$
c_{m}\left(\beta_{n m}\right)=2 \cos m \alpha_{n m}-2=0
$$

from which it follows that $\beta_{n m} \equiv 2 \cos \alpha_{n m}$ is an algebraic integer. An immediate consequence is that, if $\beta_{n m}$ is a rational number, it must be a rational integer. Note that its MP is not $c_{m}(x)$, but a factor of $c_{m}(x)$, as discussed above in item 3 . Hence, any conjugate of $\beta_{n m}$ must also be a root of $c_{m}(x)$.

Next, except for $m=1$ or 2 , the algebraic degree of $\beta_{n m}$ is given by $\varphi(m) / 2$ where $\varphi(m)$ is Euler's $\varphi$-function [24], i.e.,

$$
\operatorname{deg}\left(2 \cos \alpha_{n m}\right)=\frac{\varphi(m)}{2}=\frac{m}{2} \prod_{P \mid m}\left(1-P^{-1}\right)
$$

where $P$ are prime numbers and the product involves all the distinct prime factors of $m$. Recall that, by definition, $\varphi(m)$ is equal to the the number of positive integers that are less than $m$ and are relatively prime to $m$. A useful property of $\varphi(m)$ is that $\varphi(m)$ tends to increase with $m$. In fact, various lower bounds on $\varphi(m)$ can be established [25]. For instance, $\varphi(m) \geq \sqrt{m}$ for $m \neq 2$ and $m \neq 6$ and $\varphi(m)>m^{2 / 3}$ for $m>30$.

A rigorous proof of Eq. (A2) can be found in [26]. A short but less elementary proof can be found in [27]. Some examples are given in table [1 of section III. Below we give a simple argument showing that the conjugates of $\beta_{n m}$, i.e., the roots of its MP, can include only those $2 \cos \alpha_{q m}$ with $\operatorname{gcd}(q, m)=1$, the number of which is exactly $\varphi(m) / 2$ after the equality between $2 \cos \alpha_{q m}$ and $2 \cos \alpha_{(m-q) m}$ is taken into account. To show that, let us consider $\beta_{k p}=2 \cos \alpha_{k p}$ and $\beta_{n m}=2 \cos \alpha_{n m}$ with $\operatorname{gcd}(k, p)=\operatorname{gcd}(n, m)=1$ and $p<m$. From $c_{p}\left(\beta_{k p}\right)=0$ and

$$
c_{p}\left(\beta_{n m}\right)=2 \cos p \alpha_{n m}-2 \neq 0
$$

one finds that $\beta_{n m}$ cannot be a root of $c_{p}(x)$ which contains the MP of $\beta_{k p}$. Therefore, $\beta_{n m}$ is not a root of the MP of any $2 \cos \alpha_{q m}$ if $q$ is not relatively prime to $m$. In other words,

\footnotetext{
${ }^{7}$ If more details are needed, note that $c_{m}(x)$ can also be written as $2 T_{n}(x / 2)-2$ where $T_{n}(x)$ are Chebyshev polynomials of the first kind 23$]$.
} 
the former is not a conjugate of the latter. Since all the conjugates of $\beta_{n m}$ are among the roots of $c_{m}(x)$ which can be written as $2 \cos \alpha_{q m}$ with $1 \leq q \leq m$, we are left with $2 \cos \alpha_{q m}$ with $\operatorname{gcd}(q, m)=1$, as promised.

Now we turn to the sum of the conjugates of $\beta_{n m}$, including all the distinct $2 \cos \alpha_{q m}$ with $\operatorname{gcd}(q, m)=1$. The results are given by

$$
\sum_{c} 2 \cos \alpha_{n m}= \begin{cases}2, & m=1 \\ -2, & m=2 \\ \mu(m), & m \geq 3\end{cases}
$$

where $\sum_{c} \theta$ denotes the sum of the conjugates of an algebraic number $\theta$ and $\mu(m)$ is the Möbius function [24] defined as

$$
\mu(m)= \begin{cases}1, & m=1 \\ 0, & \text { if } m \text { has a square factor } \\ (-1)^{k}, & \text { if } m=p_{1} p_{2} \ldots p_{k} \text { with } p_{i} \text { being different prime numbers }\end{cases}
$$

For some examples, see table $\llbracket$ in section III. Below we give an example to demonstrate how Eq. (A3) is derived. First, one has

$$
\sum_{k=1}^{m-1} \cos \frac{k}{m} 2 \pi=\operatorname{Re}\left[\sum_{k=1}^{m-1} e^{i \frac{k}{m} 2 \pi}\right]=-1
$$

for any $m \geq 2$. Then one can divide the terms on the left-hand side into groups of conjugates. For example, for $m=3 \times 7$,

$$
\sum_{k=1}^{20} \cos \frac{k}{21} 2 \pi=\sum_{i=1}^{2} \cos \frac{i}{3} 2 \pi+\sum_{i=1}^{6} \cos \frac{i}{7} 2 \pi+2 \sum_{\substack{j \leq 10,(j, 21)=1}} \cos \frac{j}{21} 2 \pi
$$

where $(j, 21)$ is short for $\operatorname{gcd}(j, 21)$. As explained above, the last term can be written as $\sum_{c} 2 \cos (2 \pi n / 21)$ where $n$ can be any integer satisfying $\operatorname{gcd}(n, 21)=1$. From Eq. (A4) and (A5) one finds that

$$
\sum_{c} 2 \cos \frac{n}{21} 2 \pi=1
$$

By induction the result given by Eq. (A33) can also be proved for arbitrary $m$ in a similar way. The detailed derivation is somewhat lengthy and hence will not be presented here. In addition, for a result like Eq. (A3), it seems to us that numerical verification might be 
a simple way to convince oneself. We have done that for $m \leq 5 \times 10^{4}$, which should be sufficient for us because in our discussions $m$ represents the order of a group element.

[1] C. S. Lam, Phys. Rev. Lett. 101, 121602 (2008) arXiv:0804.2622];

[2] C. S. Lam, Phys. Rev. D 78, 073015 (2008) arXiv:0809.1185].

[3] W. Grimus, L. Lavoura and P. O. Ludl, J. Phys. G 36, 115007 (2009) arXiv:0906.2689];

[4] R. de Adelhart Toorop, F. Feruglio and C. Hagedorn, Phys. Lett. B 703, 447 (2011) arXiv:1107.3486.

[5] R. de Adelhart Toorop, F. Feruglio and C. Hagedorn, Nucl. Phys. B 858, 437 (2012) arXiv:1112.1340.

[6] D. Hernandez and A. Yu. Smirnov, Phys. Rev. D 86, 053014 (2012) [arXiv:1204.0445].

[7] D. Hernandez and A. Yu. Smirnov, Phys. Rev. D 87, 053005 (2013) arXiv:1212.2149].

[8] B. Hu, Phys. Rev. D 87, 033002 (2013) arXiv:1212.2819.

[9] W. Grimus, J. Phys. G 40, 075008 (2013) arXiv:1301.0495].

[10] C. S. Lam, Phys. Rev. D 87, 053018 (2013) [arXiv:1301.3121].

[11] D. Hernandez and A. Yu. Smirnov, Phys. Rev. D 88, 093007 (2013) arXiv:1304.7738.

[12] R. M. Fonseca and W. Grimus, JHEP 1409, 033 (2014) arXiv:1405.3678].

[13] S.-F. Ge, H.-J. He and F.-R. Yin, JCAP 1005, 017 (2010) [arXiv:1001.0940]; S.-F. Ge, D. A. Dicus, and W. W. Repko, Phys. Lett. B 702, 220 (2011) arXiv:1104.0602] H.-J. He and F.-R. Yin, Phys. Rev. D 84, 033009 (2011) arXiv:1104.2654]; S.-F. Ge, D. A. Dicus, and W. W. Repko, Phys. Rev. Lett. 108, 041801 (2012) arXiv:1108.0964]; H.-J. He and X.-J. Xu, Phys. Rev. D 86, 111301 (2012) arXiv:1203.2908; A. D. Hanlon, S.-F. Ge, and W. W. Repko, Phys. Lett. B 729, 185 (2014) arXiv:1308.6522]; A. D. Hanlon, W. W. Repko and D. A. Dicus, Adv. High Energy Phys. 2014, 469572 (2014) arXiv:1403.7552]; S.-F. Ge, arXiv:1406.1985.

[14] G. Altarelli and F. Feruglio, Rev. Mod. Phys. 82, 2701 (2010) arXiv:1002.0211]; H. Ishimori, et al., Prog. Theor. Phys. Suppl. 183, 1 (2010) arXiv:1003.3552]; W. Grimus and P. O. Ludl, J. Phys. A 45, 233001 (2012) arXiv:1110.6376]; S. F. King and C. Luhn, Rept. Prog. Phys. 76, 056201 (2013) arXiv:1301.1340]; S. F. King, et al., New J. Phys. 16, 045018 (2014) arXiv:1402.4271.

[15] N. Memenga, W. Rodejohann and H. Zhang, Phys. Rev. D 87, 053021 (2013) 
arXiv:1301.2963, P. M. Ferreira, L. Lavoura and P. O. Ludl, Phys. Lett. B 726, 767 (2013) arXiv:1306.1500; G. -J. Ding, S. F. King and A. J. Stuart, JHEP 1312, 006 (2013) arXiv:1307.4212]; I. de Medeiros Varzielas and L. Lavoura, J. Phys. G 41, 055005 (2014) arXiv:1312.0215.

[16] C. S. Lam, Phys. Rev. D 87, 053012 (2013) arXiv:1301.1736]; S. F. King, T. Neder and A. J. Stuart, Phys. Lett. B 726, 312 (2013) arXiv:1305.3200]; C. C. Nishi, Phys. Rev. D 88, 033010 (2013) [arXiv:1306.0877]; I. Medeiros Varzielas and D. Pidt, JHEP 1311, 206 (2013) arXiv:1307.6545]; T. Araki, H. Ishida, H. Ishimori, T. Kobayashi, and A. Ogasahara, Phys. Rev. D 88, 096002 (2013) arXiv:1309.4217]; S. F. King and T. Neder, Phys. Lett. B 736, 308 (2014) arXiv:1403.1758; H. Ishimori and S. F. King, arXiv:1403.4395; G. -J. Ding and S. F. King, Phys. Rev. D 89, 093020 (2014) arXiv:1403.5846]; G. -J. Ding and Y. -L. Zhou, JHEP 1406, 023 (2014) arXiv:1404.0592]; P. F. Harrison, R. Krishnan and W. G. Scott, Int. J. Mod. Phys. 29, 1450095 (2014) arXiv:1406.2025.

[17] P. S. Bhupal Dev, R. N. Mohapatra and M. Severson, Phys. Rev. D 84, 053005 (2011) arXiv:1107.2378]; P. S. Bhupal Dev, B. Dutta, R. N. Mohapatra and M. Severson, Phys. Rev. D 86, 035002 (2012) arXiv:1202.4012]; F. Feruglio, C. Hagedorn and R. Ziegler, Eur. Phys. J. C 74, 2753 (2014) arXiv:1303.7178; G. -J. Ding, S. F. King, C. Luhn and A. J. Stuart, JHEP 1305, 084 (2013) arXiv:1303.6180]; G. J. Ding and Y. L. Zhou, Nucl. Phys. B 876, 418 (2013) arXiv:1304.2645] C. Luhn, Nucl. Phys. B 875, 80 (2013) arXiv:1306.2358; Y. Kajiyama, H. Okada and K. Yagyu, Nucl. Phys. B 887, 358 (2014) arXiv:1309.6234; Y. Zhao and P. -F. Zhang, arXiv:1402.5834.

[18] C. Liu, Commun. Theor. Phys. 47, 1088 (2007) hep-ph/0507298; B. Hu, F. Wu, and Y.L. Wu, Phys. Rev. D 75, 113003 (2007) hep-ph/0612258]; A. Aranda, C. Bonilla and A. D. Rojas, Phys. Rev. D 85, 036004 (2012) [arXiv:1110.1182]; C. Liu and Z.-h. Zhao, arXiv:1205.3849; S. Gupta, A. S. Joshipura and K. M. Patel, JHEP 1309, 035 (2013) arXiv:1301.7130] A. S. Joshipura and K. M. Patel, Phys. Lett. B 727, 480 (2013) arXiv:1306.1890]; M. Holthausen and K. S. Lim, Phys. Rev. D 88, 033018 (2013) arXiv:1306.4356]; Y. Kajiyama, H. Okada and K. Yagyu, JHEP 1310, 196 (2013) [arXiv:1307.0480]; C. Hagedorn, A. Meroni and L. Vitale, J. Phys. A 47, 055201 (2014) arXiv:1307.5308]; W. Grimus and L. Lavoura, JHEP 1403, 004 (2014) arXiv:1309.3186]; J. Kile, M. J. Pérez, P. Ramond and J. Zhang, JHEP 1402, 036 (2014) arXiv:1311.4553]; J. C. Gómez-Izquierdo, F. González Canales and M Mondragon, 
arXiv:1312.7385; A. S. Joshipura and K. M. Patel, JHEP 1404, 009 (2014) arXiv:1401.6397];

R. Krishnan, arXiv:1402.0857; C. S. Lam, arXiv:1403.7835; H. Abe, T. Kobayashi, H. Ohki, K. Sumita and Y. Tatsuta, JHEP 1406, 017 (2014) arXiv:1404.0137]; A. S. Joshipura and K. M. Patel, Phys. Rev. D 90, 036005 (2014) arXiv:1405.6106].

[19] GAP - Groups, Algorithms, Programming - A System for Computational Discrete Algebra, http://www.gap-system.org.

[20] C. S. Lam, Phys. Rev. D 87, 013001 (2013) arXiv:1208.5527]; M. Holthausen, K. S. Lim and M. Lindner, Phys. Lett. B 721, 61 (2013) arXiv:1212.2411]; L. Lavoura and P. O. Ludl, Phys. Lett. B 731, 331 (2014) arXiv:1401.5036.

[21] J. H. Conway and A. J. Jones, Acta Arithmetica 30, 229 (1976).

[22] H. Pollard and H. G. Diamond, The Theory of Algebraic Numbers, 3rd, rev. edition (Dover Publications, 1998).

[23] See, for example, I. S. Gradshteyn and I. M. Ryzhik, Table of Integrals, Series, and Products, 7th edition (Academic Press, 2007).

[24] G. H. Hardy and E. M. Wright, An Introduction to the Theory of Numbers, 6th edition (Oxford University Press, 2008).

[25] See, for example, D. S. Mitrinović, J. Sándor, and B. Crstici, Handbook of Number Theory I (Springer, 2006), and references therein.

[26] I. Niven, Irrational Numbers (The Mathematical Association of America, 1956).

[27] W. Watkins and J. Zeitlin, The American Mathematical Monthly 100, 471 (1993).

[28] B. Poonen and M. Rubinstein, SIAM Journal on Discrete Mathematics 11, 135 (1998). 